This document is the accepted manuscript version of the following article:

Smolentsev, G., Soldatov, M. A., Probst, B., Bachmann, C., Azzaroli, N., Alberto, R., ... van Bokhoven, J. A. (2018). Structure of the CoI intermediate of a cobalt pentapyridyl catalyst for hydrogen evolution revealed by time-resolved X-ray spectroscopy. Chemsuschem, 11(18), 3087-3091.

\title{
Structure of the Co' intermediate of a cobalt pentapyridyl catalyst for hydrogen evolution revealed by time-resolved X-ray
} spectroscopy

\author{
Grigory Smolentsev, ${ }^{*[a, b]}$ Mikhail A. Soldatov,${ }^{[b]}$ Benjamin Probst,${ }^{[c]}$ Cyril Bachmann, ${ }^{[c]}$ Nicolo Azzaroli, ${ }^{[a]}$ \\ Roger Alberto, ${ }^{[\mathrm{c}]}$ Maarten Nachtegaal, ${ }^{[\mathrm{a}]}$ and Jeroen A. van Bokhoven ${ }^{[\mathrm{a}, \mathrm{d}]}$
}

\begin{abstract}
Cobalt polypyridyls are highly efficient water-stable molecular catalysts for hydrogen evolution. The catalytic mechanism explaining their activity is under debate and the main question is the involvement of pyridyls in the proton transfer: the pentapyridyl ligand, acting as a pentadentate ligand, can provide stability to the catalyst or one of the pyridines can be involved in the proton transfer. Using time-resolved Co $\mathrm{K}$-edge X-ray absorption spectroscopy in the microsecond time range, we have found that for the Co"(aPPy) catalyst $($ aPPy $=$ di $([2,2$ '-bipyridin $]-6-y l)($ pyridin-2-yl)methanol) the pendant pyridine dissociates from the cobalt in the intermediate $\mathrm{Co}^{\prime}$ state. This opens the possibility for the pyridinium to act as an intramolecular proton donor. In the resting state, the catalyst returns to the original six-coordinated high spin Co" state with a pentapyridyl and one water molecule coordinating the metal center. Such a bifunctional role of the polypirdyl ligands can be exploited during further optimization of the catalyst.
\end{abstract}

The storage of solar energy in chemical bonds, for example in molecular hydrogen, is a promising strategy to reduce fossil fuel usage. ${ }^{[1-3]}$ Hydrogen-evolving catalysts based on $3 d$ elements cobalt, nickel and iron attract special attention ${ }^{[4-9]}$, because they are cheap, earth-abundant and therefore promising for largescale applications. Molecular photocatalytic systems provide flexibility in the design of efficient catalysts by modification of the organic ligands. The rational design of such molecular catalysts requires a detailed knowledge of the catalytic mechanism, which is the basis for understanding the relationship between structure and performance.

The most-studied molecular catalysts for hydrogen evolution are cobaloximes. ${ }^{[4]}$ They can be combined with different photosensitizers and electron donors in organic solvents and their mixtures with water. The highest reported turnover number (TON) for cobaloximes is around 2000 hydrogen molecules per cobalt atom ${ }^{[10,11]}$. The advantage of cobaloximes is the low overpotential required $(\sim 200 \mathrm{mV})^{[4]}$. Unfortunately, cobaloxime catalysts are not very stable in water and degradation of the catalyst due to nanoparticle formation was reported in

\footnotetext{
[a] Dr. G. Smolentsev, Dr. N. Azzaroli, Dr. M. Nachtegaal and Prof. J.A. van Bokhoven

Paul Scherrer Institute, Villigen, 5232, Switzerland grigory.smolentsev@psi.ch

[b] Dr. G. Smolentsev, Dr. M.A. Soldatov

Smart Materials International Research Center, Southern Federal University of Russia, Rostov-on-Don, 344090, Russian Federation

[c] Dr. B. Probst, Dr. C. Bachmann, Prof. R. Alberto Department of Chemistry,

University of Zurich, Zurich, 8057, Switzerland

[d] Prof. J.A. van Bokhoven

ETH Zurich, Zurich 8093, Switzerland
}

Supporting information for this article is given via a link at the end of the document electrochemical assays ${ }^{[12]}$. The large-scale application of solar fuel technology requires systems that work in water. During the last six years, cobalt polypyridyls have appeared as a new family of water stable, hydrogen-evolving catalysts. A TON of $30^{\prime} 000^{[13]}$ and turnover frequency (TOF) of $10^{\prime} 000\left(\mathrm{~h}^{-1}\right)$ have been achieved $^{[14]}$ for a system based on Co"(aPPy) (structure is shown at Figure 1). Such progress was possible due to an empirical search for optimal ligands and conditions by various research groups. ${ }^{[14-20]}$

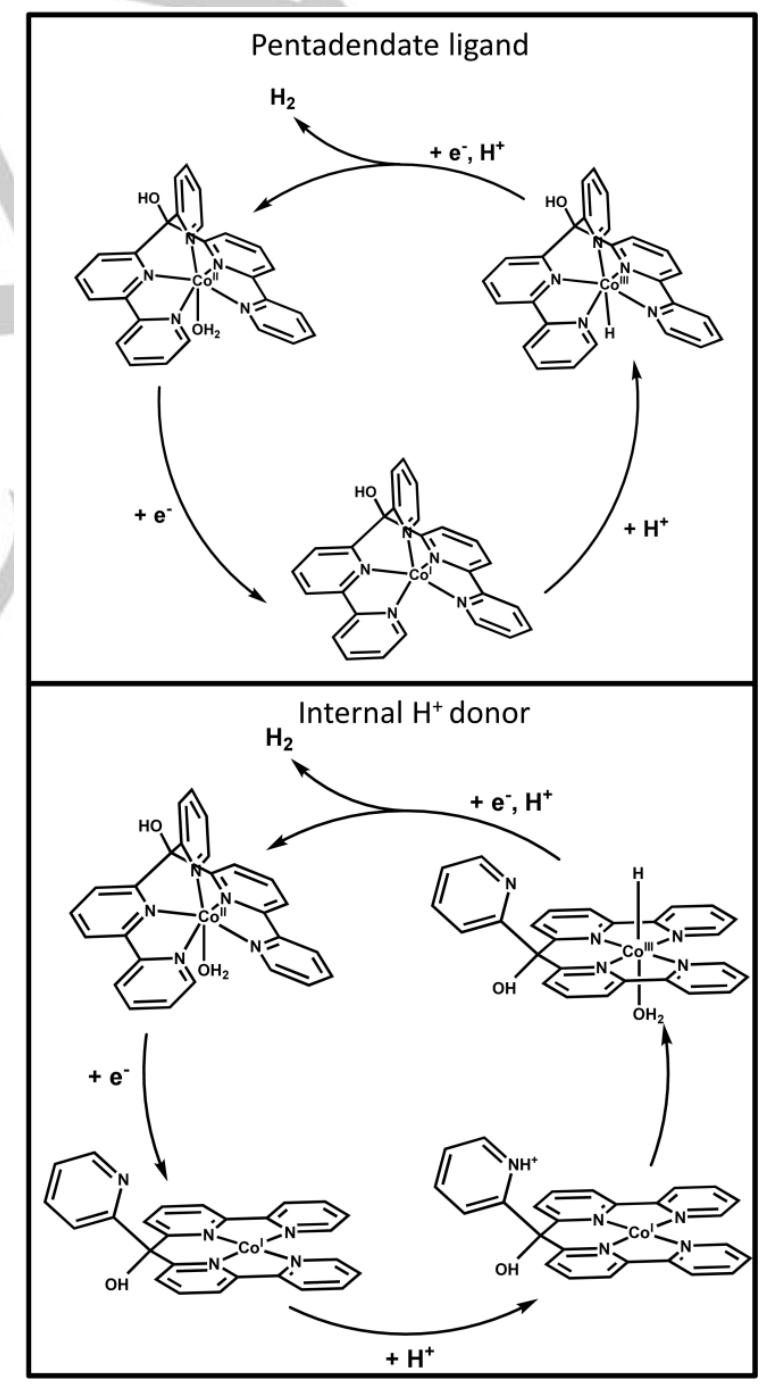

Scheme 1. Photocatalytic hydrogen evolution in a multicomponent photocatalytic system with the Co"(aPPy) catalyst. Top panel: mechanism in which aPPy is a pentadentate ligand; Bottom panel: mechanism with dissociated pyridine acting as internal proton donor. 
The Co"(aPPy) catalyst has a pentadentate polypyridyl ligand. In the initial Co" state (Scheme 1) in water, metal is coordinated by the ligand, consisting of the two bpy (2,2'-bipyridine-6-yl) subunits and one single py (pyridin-2-yl), which are bridged by a methanol group and a solvent water molecule at the sixth site (Figure 1). This structure has been obtained using a DFT optimation starting from X-ray diffraction data reported previously ${ }^{[14]}$. One possible mechanism of hydrogen evolution by the Co"(aPPy) catalyst is analogous to the mechanism suggested for cobaloxime ${ }^{[21,22]}$ and cobalt tetrapyridyl complexes $^{[20,23]}$. In this mechanism the first intermediate is a Co


(Scheme 1, top). Further reaction paths are under debate in the literature and depend on the precise experimental conditions, such as catalyst concentration, the kind of solvent and $\mathrm{pH}$. For high catalyst concentrations, the homolytic path is possible ${ }^{[22,24,25]}$ with two $\mathrm{Co}^{111} \mathrm{H}$ molecules releasing hydrogen and returning to the Co" state. In the case of a low catalyst concentration and high concentration of protons the heterolytic path is possible with the protonation of the cobalt-hydride bond and the cobalt in the $+\mathrm{II}$ or + III state ${ }^{[21,22,26]}$. An important feature of this mechanism is the presence of only one protonation site localized at the cobalt center.

Another type of catalytic mechanism used in molecular hydrogen evolving catalysts, which could potentially be used to describe hydrogen evolution by the Co"(aPPy) catalyst, originates from the analogy with $\mathrm{FeFe}$ and NiFe hydrogenases ${ }^{[27-29]}$. The key feature here is an intramolecular proton donor located in close proximity to the metal center. In a nickel molecular catalyst developed by DuBois, a pendant amine acts as the proton donor while the hydride is located on the nickel ${ }^{[30-32]}$. If pyridine dissociates from the metal (Scheme 1, bottom), pyridinium can form at later reaction steps, and potentially function as the proton donor $\left(\mathrm{pH}\right.$ reaction $=5 ; \mathrm{pK}_{\mathrm{a}}$ pyridine $\left.5.25^{[33]}\right)$. Proton transfer is a step with a large energy barrier. Therefore, optimization of this step by placing the proton donor at the right distance from the metal center, corresponding to the minimal energy barrier, is a promising approach to the rational design of hydrogen-evolving catalysts.

Similar mechanisms involving the dissociation of the metalpyridine bond were suggested for a cobalt catalyst with a pentadentate ligand bearing four pyridines and a tertiary, bridging amine ${ }^{[34]}$ and for nickel pyridinethiolate complexes ${ }^{[35]}$. Arguments favoring the dissociation of pyridine originate from electro-catalytic studies and ${ }^{1} \mathrm{H}$ NMR, but are not supported by direct structural evidence. Therefore, a key question that needs to be addressed is: does pyridine dissociate from cobalt after the first reduction step? If it dissociates, then, potentially, the pyridinium can act as intramolecular proton donor thereby enhancing the catalytic activity (Scheme 1, bottom). The formation of a penta-coordinated Co' complex with a solvent molecule localized between pyridine and cobalt can block this mechanism. In the case of a penta-coordinated $\mathrm{Co}^{\prime}$ intermediate either with pyridine or with water axially coordinating cobalt, the only possible mechanism (Scheme 1, top) is similar to those suggested for cobaloxime with protonation of the cobalt site.

Co K-edge X-ray absorption near edge structure (XANES) spectra are uniquely sensitive to the local atomic structure around the cobalt atom and its oxidation state. Time-resolved XANES spectroscopy extends the possibilities of the method from species observable at steady-state conditions to excited states and transiently formed intermediates of photochemical reactions ${ }^{[36-40]}$. The microsecond range is most relevant to study intermediates of catalysts in multicomponent photocatalytic systems $^{[41-46]}$. Thus, the time-resolved XANES enables the investigation of the structure of the cobalt photo-catalytic intermediate. Time-resolved XAS measurements were performed using pump-sequential-probes setup at the SuperXAS beamline of the Swiss Light Source ${ }^{[41]}$. A laser with $447 \mathrm{~nm}$ wavelength was used to excite the photosensitizer. A series of X-ray pulses following after the optical pulse allowed to measure the kinetics and the spectroscopic information in the time range between $20 \mathrm{~ns}$ and $50 \mu \mathrm{s}$ (see SI).

We measured time-resolved XAS in the microsecond range on solutions consisting of the photosensitizer $\left[\mathrm{Ru}(\text { bipy })_{3}\right] \mathrm{Cl}_{2}$ (PS, $0.5 \mathrm{mM}$ ), the water reduction catalyst $[\mathrm{CoBr}(\mathrm{appy})] \mathrm{Br}$ (WRC, $0.8 \mathrm{mM}), 100 \mathrm{mM}$ ascorbic acid, $100 \mathrm{mM}$ tris(2-carboxyethyl) phosphine hydrochloride (TCEP), $\mathrm{pH}=5$ or 6 , in $\mathrm{H}_{2} \mathrm{O}$. Photocatalytic activity of such a system and its characterization with time-resolved optical spectroscopy have been reported previously ${ }^{[4]}$. In comparison to widely used systems with PS, WRC and ascorbic acid the additional use of TCEP prevents accumulation of dehydroascorbic acid (DHA), which otherwise affects the lifetime of the reduced intermediates when accumulated. In other words TCEP makes the photocatalytic system more stable at the hours time scale.

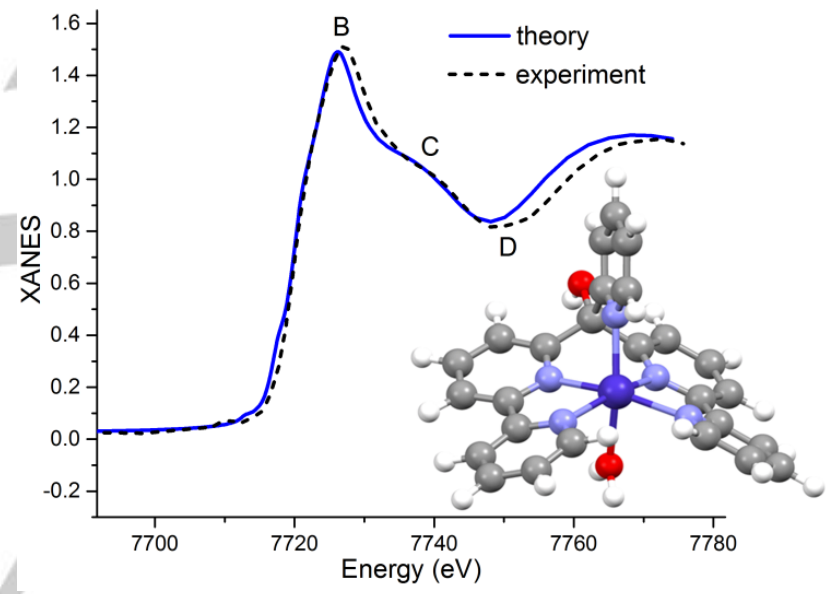

Figure 1. Comparison of experimental and theoretical XANES spectra of Co"(aPPy) in the initial state in water obtained for DFT-optimized structure. The concentration of Co"(aPPy) is $0.8 \mathrm{mM}$. Insert shows the DFT-optimized structure. $\mathrm{C}$ atoms are grey, $\mathrm{H}$ white, $\mathrm{O}$ red, $\mathrm{N}$ light blue, Co dark blue. Two $\mathrm{Br}$ ions, which are present in the crystal structure ${ }^{[14]}$, do not coordinate to the metal in solution and are therefore not shown.

The Co K-edge XANES of the initial Co" state is shown in Fig 1 and the transient spectrum, the difference between spectra of intermediate and initial states, is presented in Fig. 2 and Fig S1. For a reliable comparison of the different structural models of the intermediate, it is important to achieve good agreement between theory and experiment for the initial state XANES spectrum. We have constructed a model of the Co"(aPPy) in solution using structural optimization based on DFT calculations (Fig 1 insert). The XRD structure ${ }^{[14]}$ is the starting point for the optimization, with the replacement of bromide by water. A solvent molecule can be placed in the cis or trans position relative to the single pyridine ligand. Spectra for these two models are similar (Fig S2), but the calculated XANES spectrum for the trans structure reveals a slightly better agreement with the experimental one. Also, the high spin configuration of Co" shows a much better overlap with the experiment than the low spin configuration (Fig S3), in agreement with the results of SQUID measurements ${ }^{[48]}$. 
To double-check distances in the first coordination shell of cobalt, we have performed the EXAFS measurements and analysis for the catalyst in solution (Fig S4). The average Co-N bond length in the DFT model with trans solvent position is $2.13 \AA$, which is in agreement with the fitted value from EXAFS $(2.12 \AA)$ and close to the crystallographic one $(2.15 \AA)$. The calculated XANES of the Co"(aPPy) agrees with the experimental spectrum (Fig. 1).

The electron transfer from reduced PS to Co" occurs on a time scale of about $1 \mu \mathrm{s}$ (Fig S5), which is consistent with rate constants reported in the literature. ${ }^{[15,23]}$ The transient XANES spectrum (the difference between the spectra of the intermediate and initial state) corresponding to the $10 \mu \mathrm{s}$ time window after the excitation is shown in Fig 2. The shape of transient does not change significantly within the considered time interval from -25 to $25 \mu \mathrm{s}$ (Fig S6). It was confirmed by the presence of two statistically significant components (ground state and one intermediate) using the principal component analysis approach described previously ${ }^{[49]}$. The transient spectrum has a small positive peak $A^{\prime}$ at energy $7716 \mathrm{eV}$, which corresponds to the rising edge of the $\mathrm{Co}^{\text {"l }}$ spectrum, and a strong negative peak $\mathrm{B}^{\prime}$ at $7725 \mathrm{eV}$, corresponding to the main maximum $\mathrm{B}$ of the absorption spectrum. If structural changes in the first coordination shell are small or if the reduction leads to bond elongation, then the absorption edge shifts to lower energies due to the changes of the oxidation state from $\mathrm{Co}^{\prime \prime}$ to $\mathrm{Co}^{\prime}$. In this case one can expect a strong positive peak of the transient spectrum at the absorption edge position. This we observed for cobaloxime that has a low spin $\mathrm{Co}^{\prime \prime}$ in the initial state ${ }^{[43]}$. Cobalt has a high spin configuration in Co"(aPPy), which leads to relatively long bonds between cobalt atom and the ligands. The Co- $\mathrm{N}$ bonds contract after cobalt reduction, which shifts the absorption maximum B to higher energy. Additionally, a lower coordination number for the $\mathrm{Co}^{\prime}$ intermediate reduces the amplitude of spectral features, in particular the intensity of the maximum B. As a result, a strong negative peak B' at the position of the absorption maximum $B$ is observed in the transient spectrum. The interplay between the influence of the structure and shift to lower energy due to cobalt reduction defines the presence and intensity of the first positive peak A'.
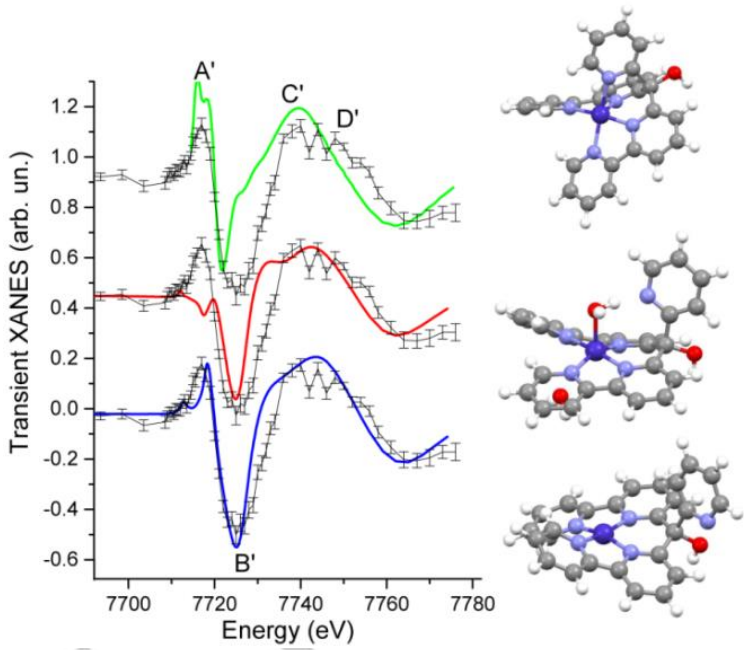

Figure 2. Experimental Co K-edge transient XANES spectrum measured at $\mathrm{pH} 6.0$, corresponding to the $10 \mu \mathrm{s}$ time window after photoexcitation (black lines) is compared with theoretical calculations for different models of the Co intermediate (shown on the right).
To clarify the structure of the active intermediate, we tested three likely DFT-optimized structural models, calculated theoretical XANES spectra of these and compared the theoretical transient XANES with the experimental data. Comparison of experimental and theoretical transient XANES spectra is more precise than the direct comparison of the XANES of the intermediate, because of the small discrepancy of theory and experiment as seen for the initial state (Figure 1) is cancelled out during the analysis of transients. We considered two penta-coordinated models of $\mathrm{Co}^{\prime}$ since this type of structure has previously been proposed for a cobaloxime hydrogen evolving catalyst, ${ }^{[2,25]}$ and a tetracoordinated structure, which is typical for the $3 d^{8}$ configuration. Transient XANES spectra for Co' models with the same type of coordination, but with different spin configurations are similar (Fig S7), therefore only the low spin case is shown in Fig. 2. In the first model (Fig. 2, top), only the dissociation of a water molecule from the cobalt center was considered, so the metal is penta-coordinated. The average Co- $\mathrm{N}$ distance contracts by $0.14 \AA$ relative to the initial Co" structure. The corresponding theoretical difference spectrum has a low energy positive peak $\left(A^{\prime}\right)$ with a higher relative intensity than experimentally observed. The first negative peak ( $\mathrm{B}^{\prime}$ ) is shifted to lower energy than in the experimental data with a position corresponding to the rising edge of the Coll state. In the second model (Fig. 2, middle), a pyridine ligand dissociates from the cobalt and is replaced by water. The cobalt-water distance is $2.16 \AA$, while the average Co-N bond length is $1.92 \AA$. In this model, the negative peak of the transient spectrum ( $B$ ') has the correct energy position and almost the right intensity, but the first positive peak $\left(A^{\prime}\right)$ is completely missing. Finally, in model 3 (Fig. 3, bottom), the cobalt center is tetra-coordinated due to the dissociation of a single pyridine from cobalt. The resulting average Co-N distance is $1.90 \AA$. In this model, all features of the transient XANES spectrum are reproduced and have the correct intensities, apart from a small shift of the first positive peak to higher energies relative to the experimental data. The pyridine group in model 3 is oriented in a way that the nitrogen atom is far from the cobalt center, but XANES has a very low sensitivity to $180^{\circ}$ rotation of the pyridine ligand and to the formation of a bond between $\mathrm{H}$ of alcohol and pyridine, therefore discrimination between such variations of model 3 requires further investigations. Nevertheless we can conclude that the tetra-coordinated model with the pyridine ligand dissociated from the cobalt gives the best agreement with the experiment.

Dissociation of a pyridine group, as suggested above, can explain some of the observed trends in an empirical search of efficient cobalt polypyridyl catalysts. Co"(aPPy) has the highest rate of $\mathrm{H}_{2}$ production at $\mathrm{pH} 5.0^{[20]}$. Below $\mathrm{pH} 5$, the effective concentration of ascorbate drops and limits the supply of electrons from the photochemical reaction. Above $\mathrm{pH} 5$, the hydrogen conversion drops significantly in line with the $\mathrm{pK}_{\mathrm{a}}$ of pyridine. Similar trends were observed for catalyst 7 from ref ${ }^{[19]}$. It also has a pyridine that potentially can dissociate. When dissociation of this group was constrained sterically by methyl groups, the activity decreased significantly. The flexibility of at least one pyridine ligand is obviously not the only requirement to achieve an efficient catalyst. Redox-active bipyridine ligands, which can participate in the charge accumulation at the catalyst, also have a positive impact on the performance of the catalyst. ${ }^{[18]}$ However, X-ray absorption spectroscopy is not sensitive to the reduction of the ligand, this 
effect can only be observed indirectly in the Co K-edge XAS spectrum through (probably small) structural changes that are induced by such reduction. Nevertheless, the compound that we studied combines redox-active bipyridines and a flexible pyridine ligand, thus combining two promising structural features.

In summary, we have found experimental and theoretical evidence by time-resolved X-ray spectroscopy of the dissociation of one pyridine from $\mathrm{Co}^{\prime}$ in the intermediate state of Co"(aPPy) hydrogen evolving catalyst. Accordingly, this coordination site at the metal center remains open and pyridine can potentially act as an intramolecular proton donor (Scheme 1) and increase the catalytic activity. Further optimization of this catalyst based on computational screening, should include tuning of the distance between cobalt and the pyridine ligand in the Co' complex or in the transition state $\mathrm{Co}^{\prime \prime \prime}-\mathrm{H} \mathrm{H}-\mathrm{py}$. Tuning the distance between proton donor and acceptor sites can be achieved by rational catalyst design, analogous to the optimization of the pendant amine position in a duBois type nickel catalysts ${ }^{[30,31]}$.

\section{Experimental Section}

Experiments have been performed at the SuperXAS beamline of the Swiss Light Source (SLS, Villigen, Switzerland). The pumpsequential-probes setup has been used for time-resolved XAS measurements ${ }^{[41]}$. Excitation of the sample was done at $447 \mathrm{~nm}$ by a Xiton IDOL laser with a repetition rate of $20 \mathrm{kHz}$, a pulse duration of $12 \mathrm{~ns}$ and output power of $1.2 \mathrm{~W}$. The size of the laser spot at the sample position was $200 * 200 \mu \mathrm{m}^{2}$.

Approximately $80 \mathrm{~mL}$ of sample was circulated in the closed cycle flow system. All samples contained the following components in water: TCEP (Tris(2-carboxethyl)phosphine hydrochloride: $0.1 \mathrm{M}$, as sacrificial electron donor, sodium ascorbate: $0.1 \mathrm{M}$, as electron relay, $\left[\mathrm{Ru}(\text { bipy })_{3}\right] \mathrm{Cl}_{2} 0.5 \mathrm{mM}$, as photosensitizer,[Co"(aPPy)Br]Br: $0.8 \mathrm{mM}$, as water reduction catalyst. Synthesis has been performed in the same way as reported in ${ }^{[13]}$. The samples were titrated with sodium hydroxide to the corresponding pH 5 (ca $0.25 \mathrm{M} \mathrm{NaOH}$ ). The solution was purged with $\mathrm{N}_{2}$ for 30 minutes before laser and X-ray irradiation to remove dissolved oxygen and was kept in $\mathrm{N}_{2}$ atmosphere and continuously purged during the measurements.

Calculations of XANES spectra have been performed using nonmuffin-tin finite difference method (FDM code ${ }^{[50]}$ ) using DFT optimized structures (ADF 2016 code $^{[51]}$ ). Further details are provided in the SI.

\section{Acknowledgements}

The work has been supported by Swiss National Centers for Competence in Research NCCR MARVEL, NCCR MUST, and Energy System Integration (ESI) platform at PSI. We thank the Ministry of Education and Science of the Russian Federation for the award of grant 16.3871.2017/4.6. Financial support from the Swiss National Science Foundation SNSF Sinergia project CRSII2 160801/1 and from the University of Zuerich Research Priority Project (URPP) Light to Chemical Energy Conversion "LightChEC" is gratefully acknowledged.
Keywords: keyword $1 \cdot$ keyword $2 \cdot$ keyword $3 \cdot$ keyword 4 • keyword 5

[1] N. Armaroli, V. Balzani, Chem. - Eur. J. 2016, 22, 32-57.

[2] D. Gust, T. A. Moore, A. L. Moore, Acc. Chem. Res. 2009, 42, 1890 1898

[3] N. S. Lewis, D. G. Nocera, Proc. Natl. Acad. Sci. 2006, 103, 1572915735.

[4] V. Artero, M. Chavarot-Kerlidou, M. Fontecave, Angew. Chem.-Int. Ed. 2011, 50, 7238-7266.

[5] N. Queyriaux, R. T. Jane, J. Massin, V. Artero, M. Chavarot-Kerlidou, Coord. Chem. Rev. 2015, 304-305, 3-19.

[6] D. Z. Zee, T. Chantarojsiri, J. R. Long, C. J. Chang, Acc. Chem. Res. 2015, 48, 2027-2036.

[7] J. R. McKone, S. C. Marinescu, B. S. Brunschwig, J. R. Winkler, H. B. Gray, Chem. Sci. 2014, 5, 865-878.

[8] M. R. Dubois, D. L. Dubois, Acc. Chem. Res. 2009, 42, 1974-1982.

[9] L. C. Sun, B. Akermark, S. Ott, Coord. Chem. Rev. 2005, 249, 16531663

[10] B. Probst, A. Rodenberg, M. Guttentag, P. Hamm, R. Alberto, Inorg. Chem. 2010, 49, 6453-6460.

[11] P. Du, J. Schneider, G. Luo, W. W. Brennessel, R. Eisenberg, Inorg Chem 2009, 48, 4952-4962.

[12] N. Kaeffer, A. Morozan, J. Fize, E. Martinez, L. Guetaz, V. Artero, ACS Catal. 2016, 6, 3727-3737.

[13] C. Bachmann, B. Probst, M. Guttentag, R. Alberto, Chem. Commun 2014, 50, 6737-6739.

[14] C. Bachmann, M. Guttentag, B. Spingler, R. Alberto, Inorg. Chem. 2013 52, 6055-6061.

[15] M. Guttentag, A. Rodenberg, C. Bachmann, A. Senn, P. Hamm, R Alberto, Dalton Trans. 2013, 42, 334-337.

[16] J. P. Bigi, T. E. Hanna, W. H. Harman, A. Chang, C. J. Chang, Chem. Commun. 2010, 46, 958-960.

[17] E. Deponti, A. Luisa, M. Natali, E. lengo, F. Scandola, Dalton Trans. 2014, 43, 16345-16353.

[18] M. Nippe, R. S. Khnayzer, J. A. Panetier, D. Z. Zee, B. S. Olaiya, M. Head-Gordon, C. J. Chang, F. N. Castellano, J. R. Long, Chem. Sci. 2013, 4, 3934-3945.

[19] R. S. Khnayzer, V. S. Thoi, M. Nippe, A. E. King, J. W. Jurss, K. A. E. Roz, J. R. Long, C. J. Chang, F. N. Castellano, Energy Environ. Sci. 2014, 7, 1477-1488.

[20] Schnidrig Stephan, Bachmann Cyril, Müller Peter, Weder Nicola Spingler Bernhard, Joliat-Wick Evelyne, Mosberger Mathias, Windisch Johannes, Alberto Roger, Probst Benjamin, ChemSusChem 2017, 10 4570-4580.

[21] J. T. Muckerman, E. Fujita, Chem. Commun. 2011, 47, 12456-12458.

[22] B. H. Solis, S. Hammes-Schiffer, Inorg. Chem. 2011, 50, 11252-11262.

[23] A. Rodenberg, M. Orazietti, B. Probst, C. Bachmann, R. Alberto, K. K. Baldridge, P. Hamm, Inorg. Chem. 2015, 54, 646-657.

[24] B. Probst, C. Kolano, P. Hamm, R. Alberto, Inorg. Chem. 2009, 48, 1836-1843.

[25] X. Hu, B. S. Brunschwig, J. C. Peters, J. Am. Chem. Soc. 2007, 129, 8988-8998.

[26] J. L. Dempsey, J. R. Winkler, H. B. Gray, J Am Chem Soc 2011, 132 16774-16776.

[27] A. Silakov, B. Wenk, E. Reijerse, W. Lubitz, Phys. Chem. Chem. Phys 2009, 11, 6592-6599.

[28] J. W. Peters, W. N. Lanzilotta, B. J. Lemon, L. C. Seefeldt, Science 1998, 282, 1853-1858.

[29] M. Frey, ChemBioChem 2002, 3, 153-160.

[30] A. D. Wilson, R. H. Newell, M. J. McNevin, J. T. Muckerman, M. Rakowski DuBois, D. L. DuBois, J. Am. Chem. Soc. 2006, 128, 358366.

[31] S. Raugei, S. Chen, M.-H. Ho, B. Ginovska-Pangovska, R. J. Rousseau, M. Dupuis, D. L. DuBois, R. M. Bullock, Chem. - Eur. J. 2012, 18 6493-6506.

[32] D. L. DuBois, Inorg. Chem. 2014, 53, 3935-3960.

[33] P. Atkins, J. de Paula, Atkins' Physical Chemistry, Oxford University Press, Oxford, New York, 2014. 
[34] J. Xie, Q. Zhou, C. Li, W. Wang, Y. Hou, B. Zhang, X. Wang, Chem. Commun. 2014, 50, 6520-6522.

[35] Z. Han, L. Shen, W. W. Brennessel, P. L. Holland, R. Eisenberg, J. Am. Chem. Soc. 2013, 135, 14659-14669.

[36] G. Smolentsev, V. Sundström, Coord. Chem. Rev. 2015, 304-305, 117-132.

[37] L. X. Chen, X. Zhang, J. V. Lockard, A. B. Stickrath, K. Attenkofer, G. Jennings, D. J. Liu, Acta Crystallogr. A 2010, 66, 240-251.

[38] L. X. Chen, X. Zhang, M. L. Shelby, Chem. Sci. 2014, 5, 4136-4152.

[39] M. Chergui, E. Collet, Chem. Rev. 2017, 117, 11025-11065.

[40] C. Bressler, M. Chergui, Chem. Rev. 2004, 104, 1781-1812.

[41] G. Smolentsev, A. A. Guda, M. Janousch, C. Frieh, G. Jud, F. Zamponi, M. Chavarot-Kerlidou, V. Artero, J. A. van Bokhoven, M. Nachtegaal, Faraday Discuss. 2014, 171, 259-273.

[42] G. Smolentsev, A. Guda, X. Zhang, K. Haldrup, E. S. Andreiadis, M. Chavarot-Kerlidou, S. E. Canton, M. Nachtegaal, V. Artero, V. Sundstrom, J. Phys. Chem. C 2013, 117, 17367-17375.

[43] G. Smolentsev, B. Cecconi, A. Guda, M. Chavarot-Kerlidou, J. A. van Bokhoven, M. Nachtegaal, V. Artero, Chem. - Eur. J. 2015, 21, 1515815162.

[44] D. Moonshiram, C. Gimbert-Suriñach, A. Guda, A. Picon, C. S. Lehmann, X. Zhang, G. Doumy, A. M. March, J. Benet-Buchholz, A Soldatov, et al., J. Am. Chem. Soc. 2016, 138, 10586-10596.

[45] Z.-J. Li, F. Zhan, H. Xiao, X. Zhang, Q.-Y. Kong, X.-B. Fan, W.-Q. Liu, M.-Y. Huang, C. Huang, Y.-J. Gao, et al., J. Phys. Chem. Lett. 2016, 7, 5253-5258.

[46] D. Moonshiram, A. Guda, L. Kohler, A. Picon, S. Guda, C. S. Lehmann, X. Zhang, S. H. Southworth, K. L. Mulfort, J. Phys. Chem. C 2016, 120 20049-20057.

[47] A. Rodenberg, M. Orazietti, M. Mosberger, C. Bachmann, B. Probst, R. Alberto, P. Hamm, ChemPhysChem 2016, 17, 1321-1328.

[48] C. Bachmann, Polypyridine Based Transition Metal Complexes for Homo- and Heterogeneous Photocatalytic Hydrogen Production from Water, PhD thesis, 2015

[49] G. Smolentsev, G. Guilera, M. Tromp, S. Pascarelli, A. V. Soldatov, J. Chem. Phys. 2009, 130, 174508.

[50] Y. Joly, Phys. Rev. B 2001, 63, 125120.

[51] G. te Velde, F. M. Bickelhaupt, E. J. Baerends, C. Fonseca Guerra, S. J. A. van Gisbergen, J. G. Snijders, T. Ziegler, J. Comput. Chem. 2001, 22, 931-967. 